\title{
Evaluation of Routine on Table Cholangiography in Paediatric Cholecystectomy
}

\author{
Roy Gurprashad", Alex Oldman, Jessica Burns, Joe Curry \\ Department of Specialist Neonatal and Paediatric Surgery, Great Ormond Street Children's Hospital, London, United Kingdom
}

Email address:

gurprashad@aol.com (R. Gurprashad)

${ }^{*}$ Corresponding author

\section{To cite this article:}

Roy Gurprashad, Alex Oldman, Jessica Burns, Joe Curry. Evaluation of Routine on Table Cholangiography in Paediatric Cholecystectomy. American Journal of Pediatrics. Vol. 3, No. 2, 2017, pp. 4-7. doi: 10.11648/j.ajp.20170302.11

Received: February 17, 2017; Accepted: March 2, 2017; Published: March 21, 2017

\begin{abstract}
The use of on table cholangiogram (OTC) during laparoscopic cholecystectomy (LC) continues to be a debated topic within surgical practice. Current National Institute for Health and Care Excellence (NICE) guidelines do not advocate its routine use in adult patients, and there is scarce evidence for its use in paediatric cases. We aimed to analyse the outcomes of OTC during laparoscopic cholecystectomy to see if the NICE guidance holds true for children. A retrospective case note review was performed with IRB approval of all children who underwent laparoscopic cholecystectomy between February 2005 and November 2014. A total of 65 patients were identified, 41 female (63\%) and 24 male (37\%). The median age was 12 years (IQR 6). None of the patients underwent OTC during their LC. Instead, pre-operative ultrasound scan (USS) was performed in all cases. From the cohort, 5 patients (13\%) showed abnormalities; 3 of which had a dilated common bile duct, and 2 of which had bile duct stones. All 5 patients went on to receive additional imaging, 2 patients underwent a repeat USS both of which were normal on review; 1 patient had an Endoscopic Retrograde Cholangio-Pancreatectography (ERCP), stent and follow-up Magnetic Resonance Cholangio-Pancreatograthy (MRCP) which was normal; 1 patient had an MRCP, ERCP with sphincterotomy and a follow-up USS which was normal; and 1 patient had an ERCP with follow-up USS which was also normal. In line with current NICE guidance for adults, our study indicates that patients with common bile duct (CBD) stones or a dilated CBD can be identified and managed prior to laparoscopic cholecystectomy. This would suggest that there is no requirement for OTC in children. Similar NICE guidance in Paediatrics may be necessary to avoid unnecessary intervention.
\end{abstract}

Keywords: On-Table-Cholangiogram, Laparoscopic, Cholecystectomy, Gallstones, Bile Duct, Guidelines

\section{Introduction}

Laparoscopic Cholecystectomy (LC) is the current best practice in the surgical management of gallstone disease in children [1] [2]; however the use of on table cholangiography (OTC) for routine LC has not been adequately investigated despite it being a frequently debated topic within adult surgical literature [3].

On table cholangiography (OTC) during LC has historically been advocated by surgeons to reduce bile duct injury, identify associated choledocholithiasis, and to avoid post-operative imaging such as Endoscopic Retrograde Cholangiopancreatogrophy (ERCP) [3]. Additionally, the ability to reduce iatrogenic morbidity through increasing anatomical definition has also been a compelling reason for adopting routine OTC during LC [4].

In opposition to routine adopters of OTC, arguments against its use include the increase in morbidity associated with additional anaesthetic time, increased procedure time and heightened departmental costs [3] [5]. Recently, two comprehensive systematic reviews have demonstrated that LC without routine requirement for OTC can be performed safely and effectively, whilst reducing total peri-operative complication [6] [7].

The LC approach developed for adult surgery has now been well adopted by paediatric surgeons in the UK, with retrospective studies strongly advocating its use over open cholecystectomy [8-13]. LC is now chosen to minimise use of analgesics, reduce complications and to improve cosmesis [1] [9].

Gallstone complications are uncommon in children, with a 
majority of patients often having underlying haemolytic conditions, such as spherocytosis, alpha and beta thalassaemia and sickle cell disease; all of which predispose to the formation of pigmented stones [10] [11]. Cholelithiasis is now no longer exceptional amongst children, with increasing obesity causing a rise in the incidence of cholesterol stones, most notably in obese adolescent girls [8]. Children on long-term total parenteral nutrition (TPN) are another welldocumented group who are at risk of biliary sludge and stones that may require surgical intervention [14] [15].

As paediatric LC has become the gold standard for gallbladder disease, there is a current paucity of paediatric LC with OTC studies. Therefore it is timely to bring the OTC debate to paediatric surgery in order to evaluate whether current guidelines, which recommend a selective approach to OTC during LC in adults, holds true for the paediatric population. The following presents a retrospective case note review of all children who underwent cholecystectomy between February 2005 and November 2014 at Great Ormond Street Children's Hospital to look at this question.

\section{Methods}

A list of all patients that underwent open or laparoscopic cholecystectomy with or without bile duct exploration was generated from the clinical coding department at Great Ormond Street Children's Hospital for the period February 2005 to November 2015. The list included patient identification numbers (PIN), primary procedure and primary procedure date.

A retrospective case note review was performed. The notes were accessed from the Electronic Document Management System (EDM). Clinic letters, operation notes and discharge summaries were identified, cross-referenced with the Patient Information Management System and Centricity PACS Web $\subset$, to ensure each patients history, examination, investigations and pathology were reviewed. Pre-operative surgical assessment data was extracted along with all preoperative and post-operative radiological procedures. Surgical and pathological findings were documented and assessed, along with post-operative complications. All patients who had an open cholecystectomy were excluded. The data was inputted onto a pre-determined Microsoft ${ }^{\mathrm{TM}}$ Excel Spreadsheet.

The project and data collection was conducted with IRB approval and registered with the trust Audit department.

\section{Results}

In total 65 patients were identified, 41 female $(63 \%)$ and 24 male (37\%). The median age was 12 years (IQR: 6) (table 1). Pain was the main presenting complaint occurring in 51 (78\%) patients. Resolution of pain on follow-up was documented in $40(62 \%)$ patients. Post-op complications were infrequent occurring in only 2 patients $(3 \%)$, one having post-operative pancreatitis and the other a gallbladder fluid collection.
Table 1. Demographics of patient cohort.

\begin{tabular}{ll}
\hline Demographic Characteristics & Frequency (\%) \\
\hline Total number of patients & $65(100)$ \\
Male & $24(37)$ \\
Female & $41(63)$ \\
Median age & 12 \\
Pancreatitis present & $6(9)$ \\
Family history of gallstones & $15(23)$ \\
Pre-operative pain & $51(78)$ \\
Post-operative pain & $11(17)$ \\
\hline
\end{tabular}

Preoperative liver function tests, (ALT and ALP), were abnormally raised in 22 patients, 9 of these patients were jaundiced. Prior to surgery, all patients underwent a preoperative Ultrasound Scan (USS). From the pre-operative USS, 3 patients had a dilated common bile duct (CBD) and 2 had bile duct stones. Pre-operative imaging performed on these 5 patients included: 2 patients underwent repeat USS which were normal on review; 1 patient had an ERCP, stent and follow-up MRCP which was normal, and 1 patient had an MRCP, ERCP with sphincterotomy and a follow-up ultrasound which was normal. Lastly, 1 patient had an ERCP with a follow-up USS that was also normal. The rest of the cohort had no other abnormalities on USS, additionally none of the patients underwent OTC during the LC procedure.

1.3.2 $73 \%(n=55)$ of the patient cohort displayed at least one co-morbidity, with $32 \%(\mathrm{n}=24)$ having multiple co morbidities. The most frequent co morbidities amongst the cohort were Hereditary Spherocytosis $(n=8)$, Splenomegaly $(n=3)$ and Hirschsprung's Disease $(n=2)$.

\section{Discussion}

Management of paediatric gallstones is now, like adults, commonly addressed using a laparoscopic approach to surgery. The benefits of LC over open cholecystectomy have been well established for paediatric cohorts, reducing costs, post-operative hospitalisation, complications, and analgesia use [12] [13]. As with adults, the role of OTC has now come into question, bringing with it both advocacy and opposition.

Our study results do not support the use of routine OTC (R-OTC) or selective OTC (S-OTC), as findings following initial USS were successfully managed pre-operatively in each case. In support of our study, a similar retrospective review of 18 children undergoing LC in India also abstained from any form of OTC across a 32-month period [16]. In their study OTC was not deemed necessary and therefore simply not performed. Selective use of OTC, i.e. OTC used on a case-by-case basis at the surgeon's discretion has been analysed in a retrospective study of 325 paediatric cholecystectomy cases [11]. Zeidan et al report that of the $202(62 \%)$ cases that were performed laparoscopically, only 20 patients $(9.9 \%)$ underwent OTC [11]. This supports the findings that R-OTC is indeed not a requirement for LC, suggesting that it be utilised only in cases where greater anatomical clarity is required. In both of these studies, postoperative complication rates were low, $11.1 \%(n=2)$ and $4.5 \%(n=9)$ respectively, of which none of the 343 patients 
across both studies had a common bile duct injury.

Historically, advocates of OTC during LC suggest the importance of cholangiography in detecting CBD stones and reducing major bile duct injury. Contemporary adult studies have highlighted the acceptably low rates of biliary complications in LC without resorting to OTC [17]. Hamad et al performed a retrospective analysis of 2714 adult cases, of these cases only $0.18 \% \quad(n=5)$ required surgical intervention of bile duct injury. Efficacy and safety of OTC has also been brought into question, with a decision analysis by Kharbutli et al demonstrating the increased risk of morbidity and mortality for LC following a positive OTC [18]. In a systematic review by Metcalfe et al of 4209 LC procedures in adults, the total false positive rate of OTC was encouragingly low, at $0.8 \%(n=34)$; however, the researchers suggested that R-OTC promotes the unnecessary instrumentation of patients, as $25 \%$ of asymptomatic CBD stones have been shown to pass through the sphincter of Oddi in the interim between OTC and post-operative ERCP [19]. They also note no significant difference in risk of CBD injury with and without OTC.

Centres operating on adults that have elected to perform OTC during LC procedures have based their decision on an inclusion criteria which incorporates traditional indicators for choledocholithiasis [3]. The 4 year 1,308 patient retrospective study by Tabone et al stated that indications for performing OTC included preoperative diagnosis of gallstone pancreatitis, increase in serum bilirubin and an elevated CBD of $>10 \mathrm{~mm}$. Despite the strict criteria, $86.5 \%(\mathrm{n}=230)$ of these selected patients who underwent OTC were deemed to be normal, and only $16.7 \%(\mathrm{n}=6)$ of the $36 \mathrm{CBD}$ stones were successfully cleared. These patients receiving OTC were ultimately hospitalised longer (3.4 days vs. 2.6 days) and incurred greater hospital costs.

With regard to limitations, we acknowledge that our study was conducted in a specialist tertiary centre where only 65 cases were identified. The low case number is anecdotally reported across the UK paediatric centres. To complement our study, further data is needed to add power to OTC paediatric research. This is an under-researched area, which would benefit from further data from surgical departments nationwide. This is certainly an area for further investigation.

\section{Conclusion}

In line with current NICE guidance for adults, our study indicates that paediatric patients with common bile duct (CBD) stones or a dilated $\mathrm{CBD}$ can be identified and managed prior to cholecystectomy without the need for ROTC. Similar NICE guidance in Paediatrics may be necessary to avoid unnecessary intervention and morbidity. See appendix 1.

\section{Acknowledgements}

We would like to thank the following who kindly permitted use of their data in this study: Simon Blackburn,
Kate Cross, David Drake, Paolo De Coppi, Ed Kiely, and Lewis Spitz.

\section{Appendix}

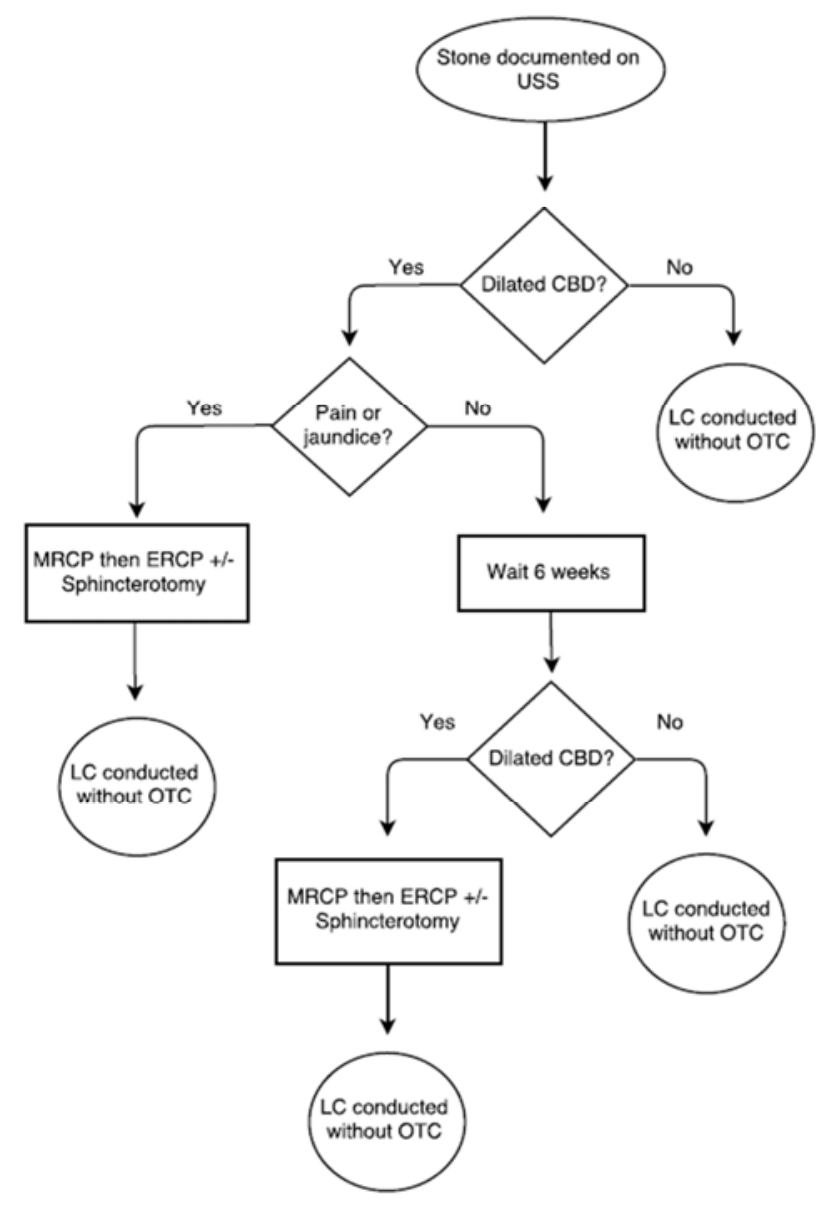

Appendix 1. Treatment algorithm.

\section{References}

[1] Chan S, Currie J, Malik A, Mahomed A. Paediatric cholecystectomy: Shifting goalposts in the laprascopic era. Surgical Endoscopy 2008; 22: 1392-1395.

[2] NICE. Single-incision laparoscopic cholecystectomy, interventional procedure guidance 508. 2014.

[3] Tabone LE, Sarker S, Fisichella M, Conlon M, Fernando E, Yi S, Luchette FA. To 'gram or not'? Indications for intraoperative cholangiogram. Surgery $2011 ; 150$ : 810-9.

[4] Sharma AK, Cherry R, Fielding JWL. A randomised trial of selective or routine on-table cholangiography. Annals of the Royal College of Surgeons of England 1993; 75: 245-248.

[5] Livingstone EH, Miller JAG, Coan B, Rege RV. Costs and utilisation of intra-operative cholangiography. The Society for Surgery of the Alimentary Tract 2007; 11: 1162-1167.

[6] Sajid MS, Leaver C, Haider Z, Worthington T, Karanjia N, Singh KK. Routine on-table cholangiography during cholecystectomy: a systematic review. Annals of the Royal College of Surgeons of England 2012; 94: 375-380. 
[7] Khan OA, Balaji S, Branagan G, Bennett DH, Davies N. Randomised clinical trial of routine on-table cholangiography during laparoscopic cholecystectomy. British Journal of Surgery 2010; 98 (3): 362-367.

[8] St Peter SD, Keckler SJ, Nair A, et al. Laparoscopic cholecystectomy in the pediatric population. J Laparoendosc Adv Surg Tech. Part A. 2008; 18: 127-130.

[9] Zeidan MM, Pandian TK, Ibrahim KA, Moir CR, Ishitani MB, Zarroug AE. Laparoscopic Cholecystectomy in the Pediatric Population: A Single-centre Experience. Surgical Laparoscopy, Endoscopy and Percutaneous Techniques 2014; 24 (3): $248-250$.

[10] Davenport M. Laparoscopic surgery in children. Annals of the Royal College of Surgeons of England 2003; 85: 324-330.

[11] Koivusalo AI, Pakarinen MP, Sittiwet C, Gylling H, Miettinen TA, Miettinen TE, Nissinen MJ. Cholesterol, non-cholesterol sterols and bile acids in paediatric gallstones. Digestive and Liver Disease 2010; 42 (1): 61-66.

[12] Kim PC, Wesson D, Superina R, Filler R. Laparoscopic Cholecystectomy Versus Open Cholecystectomy in Children: Which is Better? Journal of Paediatric Surgery 1995; 30 (7): 971-973.

[13] Jawas AJ, Kurban K, el-Bakry A, al-Rabeeah A, Seraj M, Ammar A. Laparoscopic Cholecystectomy for cholelithiasis during infancy and childhood: cost analysis and review of current indications. World J Surg 1988; 22 (1): 69-73.
[14] Pichler J, Watson T, McHugh K and Hill S. Prevalence of Gallstones Compared in Children with Different Intravenous Lipids. J Pediatr Gastroenterol Nutr 2015; 61 (20): 253-259.

[15] Colomb V, Goulet O, Rambaud C, De Potter S, Sadoun E, Ben Hariz $M$ et al. (1992). Long-term parenteral nutrition in children: liver and gallbladder disease. Transplant Proc 24, 1054-1055.

[16] Gowda DJ, Agarwal P, Bagdi R, Subramanian B, Kumar M, Ramasundaram M, Paramasamy B, Khanday ZS. Laparoscopic cholecystectomy for cholelithiasis in children. J Indian Assoc Pediatr Surg 2009; 14: 204-6.

[17] Hamad MA, Nada AA, Abdel-Atty MY, Kawashti AS. Major Biliary Complications in 2,714 cases of laparascopic cholecystectomy without intraoperative cholangiography: a multicentre retrospective study. Surg Endosc 2011; 25: 37473751 .

[18] Kharbutli B, Velanovich V. Management of preoperatively suspected choledocholithiasis: A decision analysis. Journal of Gastrointestinal Surgery; 12 (11): 1973-1980.

[19] Metcalfe MS, Ong T, Bruening MH, Iswariah H, WemyssHolden SA, Maddern GJ. Is laparascopic intraoperative cholangiogram a matter of routine? The American Journal of Surgery 2004; 187: 475-481. 